\title{
Li and CNO isotopes from magnetically induced extra-mixing in evolved stars
}

\author{
Sara Palmerini ${ }^{1,2}$, Maurizio Busso ${ }^{1,2}$, Roald Guandalini ${ }^{1}$ \\ and Enrico Maiorca ${ }^{1,2}$ \\ ${ }^{1}$ Dipartimento di Fisica, Unoverstitá degli Studi di Perugia, \\ via Pascoli, 06125, Perugia, Italy \\ email: sara.palmerini@fisica.unipg.it \\ ${ }^{2}$ I.N.F.N. sezione di Perugia, Italy
}

\begin{abstract}
Evolved low mass stars (LMS) contribute not only to the synthesis of s-process nuclei, but also to modifications in the isotopic mix of light elements ( $\mathrm{Li}$ and $\mathrm{CNO}$ especially), induced by proton captures. In particular, RGB and AGB stars show a wide range of Li abundances. This spread is currently attributed to deep phenomena of non-convective mixing. These processes can, in principle, either produce or destroy $\mathrm{Li}$, depending on their velocity. This is due to the fact that $\mathrm{Li}$ production requires preserving the unstable ${ }^{7} \mathrm{Be}$, which has a half-life of only 53 days. Physical mechanisms devised so far to explain the existence of deep mixing in low mass stars generally fail in accounting for fast transport and in avoiding ${ }^{7} \mathrm{Be}$ destruction; on the contrary, this is easily obtained in Intermediate Mass Stars, where Hot Bottom Burning can occur. However, as Li-rich low-mass red giants do exist, we propose here a scenario where both production and destruction of Li are possible in LMS, thanks to the buoyancy of magnetized parcels of processed matter, traveling from the $\mathrm{H}$ shell to the envelope at different speeds (depending on their size). Consequences of this transport for CNO nuclei are also discussed.
\end{abstract}

Keywords. Nucleosynthesis - Stars: abundances, AGB, evolution, low-mass, magnetic fields

From the original poloidal field of a rotating star, a toroidal field of similar strength can be generated by the dynamo mechanism (Parker 1974). Indeed, the differential rotation of the external layers wraps the fields, aligning them to the equator (Spruit 1999). Toroidal flux tubes then develop, with their magneto hydrodynamics instabilities, and float to the surface (Spruit \& van Ballegooijen 1982). This is so because a pressure term and a tension term (acting as an elastic force) are both created by the magnetic field. Torsion due to the Coriolis force then helps detaching portions of the tubes, or "bubbles". They move outward, with a relatively high speed, close to the Alfvén velocity (Case A; Busso et al. 2007). Due to their small size, the bubbles undergo minimal heat exchanges. When the average stellar magnetic field is strong enough to induce the buoyancy of a large portion of a flux tube, the resulting mixing velocity will be reduced (by heat exchanges) down to near the diffusion one (Case B; Palmerini \& Busso 2008, Denissenkov et al. 2009). Hence magnetic buoyancy offers a scenario for the occurrence of both fast and slow mixing episodes.

A possible scenario for the evolution of the Li abundance in low mass red giants, as a function of their bolometric magnitude, on the basis of a new estimate for their distances and evolutionary status was suggested by Guandalini et al. (2009), to which we refer for details. These authors considered a phase of magnetic field growth, at the L-bump, where field strengths were assumed to be not strong enough to promote the buoyancy of entire magnetic flux tubes, but only to generate the intermittent detaching of fast bubbles (Case A). Hence, the induced fast mixing may induce Li enrichment, as shown 


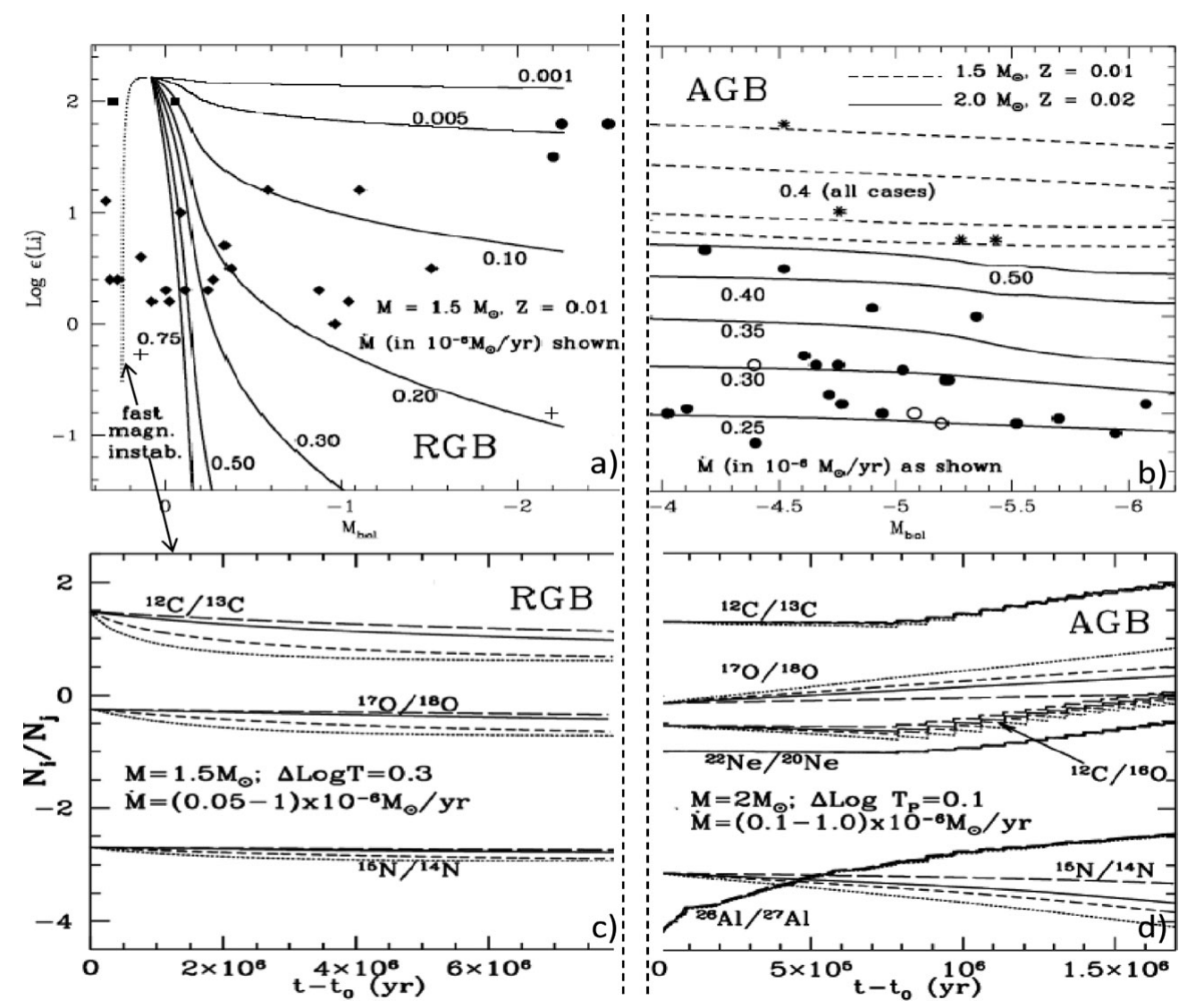

Figure 1. The evolution of $\mathrm{Li}$ (upper panels) and CNO isotopic ratios (lower panels) according to mixing cases $\mathrm{A}$ and $\mathrm{B}$, see text for details.

by several red giants in this evolutionary phase (Fig.1 panel a). When the fields have grown sufficiently, larger magnetized structures can become buoyant, driving a slower mass circulation (case B), which depletes Li during the rest of the RGB phase and the whole AGB phase (Fig.1 panel a and b). The temporal evolution of Li is accompanied by modifications of CNO isotopic ratios during both fast and slow mixing episodes. Panel c of Fig. 1 reports them at different values of the mixing rate $(\dot{M})$, for a $1.5 M_{\odot}, Z=Z_{\odot} / 2$ RGB model (Palmerini et al. 2009). Panel d instead shows temporal evolutions of CNO and $\mathrm{Al}$ isotopic ratios, in a $2 M_{\odot}, Z=Z_{\odot}$ AGB model, due to both slow mixing (case B, at different $\dot{M}$ ) and convective third dredge-up, whose contribution results in the step-wise trend of ${ }^{12} \mathrm{C} /{ }^{13} \mathrm{C},{ }^{12} \mathrm{C} /{ }^{16} \mathrm{O}$ and ${ }^{20} \mathrm{Ne} /{ }^{22} \mathrm{Ne}$ ratios.

\section{References}

Busso, M., Wasserburg, G. J., Nollett, K. M., \& Calandra, A., 2007, ApJ, 671, 802

Denissenkov, P. A., Pinsonneault, M., \& Mac Gregor K. B. 2009, ApJ, 696,1823

Guandalini, R., Palmerini, S., Busso, M., \& Uttenthaler, S. 2009, PASA, 26, 168

Palmerini, S. \& Busso M., 2008, New AR, 52, 412

Palmerini, S., Busso, M., Maiorca, E., \& Guandalini, R. 2009, PASA, 26, 161

Parker, E. N. 1974, ApESSS, 31, 261

Spruit, H. C. 1999, A\&BA, 349, 189

Spruit H. C. \& van Ballegooijen, A. 1982, A\&SA, 106, 58 